\title{
Laparoscopic Management of Ectopic Pregnancies
}

\author{
Lt Col B S Duggal*, Surg Capt Mrs P Tarneja (Retd)+, Lt Col R K Sharma", Lt Col S K Rath**, \\ Lt Col R D Wadhwa vsi ${ }^{++}$
}

\begin{abstract}
To compare the laparoscopic approach with laparotomy in the treatment of ectopic pregnancy, a retrospective analysis involving 52 patients with ectopic pregnancies was done. The aim of this study was to evaluate the safety and efficacy of laparoscopic surgery for ectopic pregnancies. 30 patients underwent laparoscopic management while 22 patients were managed by conventional laparotomy. In the laparoscopic group, the postoperative morbidity and post-hospital stay were significantly less. Although laparoscopic surgery for ectopic pregnancies is a new approach and it is not widely practised in service hospitals, it has more advantages than open surgery and it has been well accepted by the surgeons and patients. It is a safe and feasible approach.
\end{abstract}

MJAFI 2004; 60 : 220-223

Key Words : Ectopic pregnancy; Laparoscopic surgery

\section{Introduction}

A $\mathrm{n}$ ectopic pregnancy is associated with certain demise of gestation, a threat to a woman's life, and a subsequent successful pregnancy in less than $50 \%$ of patients. In our country more than $80 \%$ of ectopic pregnancies are diagnosed after rupture. With high resolution trans-vaginal sonography, serum $\beta$-HCG assay and increased vigilance of the clinician, more and more cases are being diagnosed before rupture. The incidence of ectopic pregnancy has been on the increase. In the last two decades, there has been a 6-fold increase in incidence in the United States, and a 4-fold increase in the United Kingdom [1]. Now the incidence of ectopic pregnancy is $1 \%-2 \%$ of reported pregnancies [2]. Several developments in the management of ectopic pregnancies have led to remarkable success in saving the mother's life. Further developments recently have resulted in a shift in focus from saving the mother's life to additionally saving the woman's fertility. In the past, successful surgical treatment of a tubal gestation was total salpingectomy. Since then, to improve future fertility, salpingostomy is generally adopted. Recently, laparoscopy in gynaecological surgery has developed from a diagnostic aid to a highly efficient surgical tool. Laparoscopic salpingostomy and laparoscopic salpingectomy are being performed for ectopic pregnancy. The purpose of this paper is to study these changing trends and to study the safety and efficacy of laparoscopic management of ectopic pregnancies in our hospital.

\section{Material and Methods}

A retrospective analysis involving 52 patients with ectopic pregnancy admitted between 1996 and 2001 to our hospital was carried out. The initial diagnosis of ectopic pregnancy was made through a combination of clinical examination, $\beta$ HCG assay and transvaginal ultrasonography. All patients suspected to have an ectopic pregnancy underwent a diagnostic laparoscopy. The patients were treated by laparoscopy or laparotomy based upon the haemodynamic status of the patient, experience of the surgeon and the availability of endoscopic equipment. The details of procedure, operating time and hospital stay were noted.

All the procedures were performed under general anaesthesia. Laparoscopic procedures were performed in the semilithotomy position. Through an intra-umbilical incision, trocar and cannula were introduced followed by introduction of a $10 \mathrm{~mm}$ laparoscope. After confirmation of the diagnosis, $5 \mathrm{~mm}$ punctures were made in the left and right lower quadrants using direct visualisation and transillumination to avoid the epigastric vessels. Salpingectomy was performed by stepwise desiccation of the mesosalpinx with bipolar forceps, and cutting along the mesosalpinx and across the proximal tube using scissors. For salpingostomy linear incision was given on the most prominent and distended antimesenteric border of fallopian tube with unipolar electrode knife. Products of conception were separated with fluid under pressure and sucked out. The tubal incision was left open and allowed to heal by secondary intention. Surgical specimens were generally removed through the $10 \mathrm{~mm}$ subumbilical trocar sleeve. $4 \mathrm{~mm} 30$ degree telescope (Hysteroscope) along with its sheath was introduced from side port for visualization during removal of tissue with the help of $10 \mathrm{~mm}$ grasper

${ }^{*}$ Classified Specialist (Obstetrics and Gynaecology), Military Hospital, Shillong, ${ }^{+}$Ex-Professor and Head, Department of Obstetrics and Gynaecology, Armed Forces Medical College, Pune, "Classified Specialist (Obstetrics and Gynaecology), Army Hospital (R \& R), Delhi Cantt, ${ }^{* *}$ Classified Specialist (Obstetrics and Gynaecology), 151 Base Hospital, C/o 99 APO, ${ }^{++}$Classified Specialist (Obstetrics and Gynaecology), Military Hospital, Dehradun. 
introduced from umbilical port. The pelvis was copiously irrigated with saline at the end of each procedure. Postoperative $\beta$-HCG levels were noted after six weeks in those patients who had undergone conservative procedure i.e.salpingostomy.

\section{Results}

Of the 52 patients, 30 were treated laparoscopically and 22 patients underwent laparotomy (Table-1). Out of these 22 patients, only 5 patients were haemodynamically not stable and an emergency laparotomy and salpingectomy was performed. One patient was on medical management with methotrexate and on second day had acute pain in abdomen and developed features of haemorrhagic shock. In another 7 cases laparotomy and salpingectomy had to be done due to non-availability of endoscopic equipment in emergency operation theatre although patients were haemodynamically stable inspite of sonographic evidence of tubal rupture. In 7 cases, diagnosis of unruptured tubal pregnancy was made and salpingostomy was done after opening the abdomen. 3 patients who presented as chronic ectopic, on laparotomy were found to have pelvic and para tubal haematocoele with no evidence of tubal rupture. Only saline lavage and suction was done in these cases of tubal abortion.

Table 1

Various procedures done for ectopic pregnancies

\begin{tabular}{lcc} 
Procedures & \multicolumn{2}{c}{ No. of cases done by } \\
& Laparotomy & Laparoscopy \\
Salpingostomy & 7 & 16 \\
Salpingectomy & 12 & 7 \\
$\begin{array}{l}\text { Lavage and suction of } \\
\text { pelvic haematocoele }\end{array}$ & 3 & 5 \\
Desiccation of corpus luteum & Nil & 2 \\
Total & 22 & 30
\end{tabular}

30 cases were managed laparoscopically out of which 16 salpingostomies were done. All these patients were cases of infertility who conceived after treatment or some assistance in the form of IUI. Since all these patients were under regular follow up, diagnosis of tubal pregnancy was made early. 4 patients met the criteria for medical management but in all the 4 cases medical management had to be abandoned because of patient's symptoms, non reassuring sonographic picture and treating gynaecologist's anxiety. In all these cases it was observed that bleeding was much less. In 7 cases, laparoscopic salpingectomy was done out of which 2 patients had ectopic after tubectomy done earlier. One patient had completed family and underwent laparoscopic sterilisation of other tube in addition to salpingectomy. Four patients were cases of infertility, but in 2 patients there was extensive damage due to rupture and late reporting while in another 2, tube was damaged while trying to achieve haemostasis. However, in all these cases the other tube was healthy. We had 5 cases of tubal abortion. In 3 of these cases, patients presented as chronic ectopic tubo-ovarian mass with fluid in pouch of Douglas. Laparoscopy revealed pelvic and para tubal haematocoele and adhesions with omentum, gut, and pelvic peritoneum. Adhesiolysis, hydrodissection and suction cleared the pelvis. In 2 cases, there was evidence of haematosalpinx which cleared with repeated saline lavage from fimbrial end. 2 cases presented as acute abdomen with free fluid in pouch and paracolic gutters. On laparoscopy, these patients were found to be actively bleeding from fimbrial end. Since tubes were intact and healthy, it was presumed that tubal abortion has taken place and bleeding is occuring from site of trophoblastic detachment. Repeated copious warm saline lavage could achieve haemostasis. In both these cases tube drain was left from side port which was removed after 48 hours. We had 2 cases where there was active bleeding from corpus luteum. One of these patients was a case of rheumatic heart disease with prosthetic valve on oral anticoagulants. Haemostasis could be achieved in both these cases with bipolar coagulation, however there was oozing from umbilical port in heart disease patient for 24 hours.

Average operating time for various procedures was found to be higher for laparoscopic group, however post-operative morbidity and duration of hospital stay was much less for patients managed laparoscopically (Tables 2,3 ).

\section{Discussion}

The classical surgical approach for an ectopic pregnancy is by open laparotomy. Lawson Tait first described the life saving procedure of salpingectomy by laparotomy in 1884 [3]. It was not until some 70 years later that less radical operations with conservation of the involved tube during laparotomy were performed $[4,5,6]$. Laparoscopy has been used in the diagnosis of ectopic pregnancy for many years. Since the first excision of a tubal pregnancy through a laparoscope by Manhes and Bruhat in 1980 [7], it has been used with increasing frequency in the surgical treatment of ectopic pregnancies.

Laparoscopy permits diagnosis and treatment to be combined in the same procedure and ectopic pregnancies can be diagnosed and treated at an early stage. In fact, laparoscopy is not only suitable for early ectopic pregnancies but it is also safe and feasible in instances where there is tubal rupture and hemoperitoneum, provided the patient is not severely compromised haemodynamically. In our laparoscopic group, the greatest estimated hemoperitoneum was 2,000 ml and the procedure was carried out successfully.

In our series, we have found procedures like salpingostomy, salpingectomy, peritoneal lavage and suction when done laparoscopically, had taken longer time which was found to be statistically significant. More experience with use of laparoscopic equipment and well trained nursing and paramedical staff will definitely reduce the operating time making it comparable to laparotomy.

We managed 22 cases by open laparotomy and 30 
Table 2

Comparison of operation time

\begin{tabular}{|c|c|c|c|c|c|}
\hline \multirow[t]{2}{*}{ Procedures } & \multicolumn{2}{|c|}{$\begin{array}{l}\text { Average operating time } \\
\text { (minutes) }\end{array}$} & \multirow[t]{2}{*}{$\begin{array}{c}\text { Difference } \\
\text { between means }\end{array}$} & \multirow[t]{2}{*}{ Standard error } & \multirow[t]{2}{*}{ Significance } \\
\hline & Laparotomy & Laparoscopy & & & \\
\hline Salpingostomy & 65 & 80 & 15 & 6.54 & $\mathrm{p}<0.05$ \\
\hline Salpingectomy & 60 & 70 & 10 & 5.15 & $\mathrm{p}>0.05$ \\
\hline Lavage and suction of pelvic haematocoele & 50 & 90 & 40 & 12.4 & $\mathrm{p}<0.05$ \\
\hline Desiccation of corpus luteum & - & 50 & - & - & - \\
\hline
\end{tabular}

Table 3

Comparison of duration of hospital stay

\begin{tabular}{lccccc} 
Procedures & \multicolumn{2}{c}{$\begin{array}{c}\text { Mean hospital stay } \\
\text { Laparotomy }\end{array}$} & $\begin{array}{c}\text { Laparoscopy } \\
\text { between means }\end{array}$ & Standard error & Significance \\
Salpingostomy & 7 & 2 & 5 & 0.46 & $\mathrm{p}<.001$ \\
Salpingectomy & 7 & 2 & 5 & 0.41 & $\mathrm{p}<.001$ \\
Lavage and suction of pelvic haematocoele & 7 & 3 & 4 & $0.61 \quad$ p $<.01 \quad$ r
\end{tabular}

cases by laparoscopy. Four randomised controlled trials (RCT) have reported the results of comparison between this traditional approach and laparoscopic tubal surgery [8-11]. In the paper by Murphy, there was no attempt to distinguish between salpingostomy and salpingectomy. As there was a trend towards more conservative surgery with laparoscopic approach, these results need to be interpreted with caution. In our study also, we found 12 patients underwent salpingectomy out of 22 , managed by laparotomy as compared to 7 salpingectomies out of 30 cases managed laparoscopically. Laparoscopic approach was associated with shorter hospital stay and quicker postoperative recovery time (Table 3). Yao and Tulandi [12] have shown that the subsequent intrauterine pregnancy rate was $70 \%$ after laparoscopic surgery compared to $55 \%$ after laparotomy. The recurrent ectopic rate was also lower after laparoscopy $(5 \%)$ than after laparotomy (16.6\%). However, in our small series and limitation of subsequent follow up, we had 3 intrauterine pregnancies and 2 recurrent ectopics in laparoscopy group. Patients in laparotomy group could not be followed up for subsequent pregnancies.

In 11 comparative studies [9-19], persistent trophoblast was shown to be more likely to persist after laparoscopic $(8.8 \%)$ rather than after laparotomy salpingostomy $(3.9 \%)$. We had no case of persistent trophoblastic disease out of 23 cases who had undergone salpingostomy and subsequent $\beta$-HCG follow up. In a follow up study adhesions developed significantly more often after laparotomy than after laparoscopic surgery $(\mathrm{p}<0.0001)$ [20]

In a meta-analysis of 40 studies, the intrauterine pregnancy rate after tubal conservation was $46 \%$ compared to $44 \%$ after tubal excision [21]. The repeat ectopic rate was $15 \%$ and $10 \%$ respectively. Similarly in an important meta-analysis of nine good quality comparative studies Yao and Tulandi [12] reported no significant difference in the intrauterine pregnancies between salpingostomy and salpingectomy $(53 \% \mathrm{v}$ 49.3\%). However, when these studies were diluted with other non comparative studies, $61.2 \%$ patients had intrauterine pregnancies after salpingostomy compared with only $38.1 \%$ after salpingectomy. Detailed comparison using such disparate data is not meaningful but Yao and Tulandi were able to conclude that tubal conservation may be associated with a subsequent intrauterine pregnancy rate that is at least comparable to that obtained after complete removal of the tube. The recurrent ectopic pregnancy rate was, however, higher in these studies after salpingostomy (15\%) than after salpingectomy (10\%). Failure to completely remove the ectopic occurred in $4.8 \%$ - $11 \%$ of salpingostomy cases [22-24]. In contrast, almost no cases of persistence follow salpingectomy $[25,26]$.

A single randomised trial investigated the effect of suturing the salpingostomy incision [27]. The intrauterine pregnancy rate at 12 months was higher in those without surgical repair but by 24 months the rates with or without suture were similar and it is concluded that suturing the tube after salpingostomy provides no benefit.

It is recommended that in the future all medical, nursing and support staff should be fully trained to safely undertake both the open and laparoscopic management of ectopic pregnancy. Surgeons must be experienced in operative laparoscopic techniques including the safe use of mono and bi-polar diathermy. They should also be supported with sufficient modern equipment to facilitate safe surgery. 
From literature we know that laparoscopic surgery is a more cost-effective approach. An average savings of approximately US $\$ 1,200$ - 1,500 for each patient can be obtained, because it significantly reduces the length of hospital stay.

However, the capital cost of installation of an endoscopic setup is high. In developed countries, more than $80 \%$ of ectopic pregnancies are generally treated laparoscopically. The laparoscopic approach is now the gold standard for treatment of ectopic pregnancy. We believe that conventional laparotomy will eventually be replaced by laparoscopic approach for almost all cases of ectopic pregnancies.

\section{References}

1. Fylstra DL. Tubal pregnancy: a review of current diagnosis and treatment. Obstet Gynecol Surv 1998;53:320-28.

2. Gray DT, Thorburn J, Lundorff P. A cost-effectiveness study of a randomised trial of laparoscopy versus laparotomy for ectopic pregnancy. Lancet 1995;345:1139-43.

3. Tait L. Five cases of extrauterine pregnancy operated upon at the time of rupture. BMJ 1984;1:1250-1.

4. Vehaskari A. The operation of choice for ectopic pregnancy with reference to subsequent fertility. Acta Obstet Gynecol Scand 1960;39(Suppl 3):1-41

5. Jarvinen P A. Conservative operative treatment of tubal pregnancy with post operative daily hydrotubations. Acta Obstet Gynecol Scand 1972;51:169-70.

6. Stromme W B. Conservative surgery for ectopic pregnancy: a twenty year review. Obstet Gynecol 1973;41:215-23.

7. Bruhat M A, Manhes H, Mage G, Poully J L. Treatment of ectopic pregnancy by means of laparoscopy. Fertil Steril 1980;33:411-4.

8. Murphy A A, Nager C W, Wujek J J, Kettel M M, Torp V A, Chin H G. Operative laparoscopy versus laparotomy for the management of ectopic pregnancy: a prospective trial. Fertil Steril 1992;57:1180-5.

9. Vermesh M, Silva P D, Rosen G F, Stein A L, Fossum G T, Sauer M V. Management of ruptured ectopic gestation by linear salpingostomy : a prospective randomised clinical trial of laparoscopy versus laparotomy. Obstet Gynecol 1989;73:400-4.

10. Lundorff P, Thorburn J, Hahlin M, Kallfelt B, Lindblom B. Laparoscopic surgery in ectopic pregnancy: a randomized trial versus laparotomy. Acta Obstet Gynecol Scand 1991;70:3438.

11. Konickx P R, Witters K, Brosens I, Stemers N, Oosterlynck D, Meuleman C. Conservative laparoscopic treatment of ectopic pregnancies using $\mathrm{CO}_{2}$ laser. Br J Obstet Gynecol 1991;98: 1254-9.
12. Yao M, Tulandi T. Current status of surgical and non-surgical management of ectopic pregnancy. Fertil Steril 1997;67:42133.

13. Seifer D B, Gutmann J N, Grant W D, Kamps C A, DeCherney A H. Comparison of persistent ectopic pregnancy after laparoscopic salpingostomy versus salpingostomy at laparotomy for ectopic pregnancy. Obstet Gynecol 1993;81:378-82.

14. Henderson S R. Ectopic tubal pregnancy treated by operative laparoscopy. Am J Obstet Gynecol 1989;60:1462-9.

15. Hoppe D E, Bekkar B E, Nager C W. Single-dose systemic methotrexate for the treatment of persistent ectopic pregnancy after conservative surgery. Obstet Gynecol 1994;83:51-4.

16. Keckstein G, Wolf A S, Hepp S, Layritzen C, Steiner R.Tubepreserving endoscopic surgical procedures in unruptured tubal pregnancy. What significance does laser use have? Geburtshilfe Frauenheilkunde 1990;50:207-11.

17. Brumsted J, Kessler C, Gibson C, Nakajima S, Riddick D H, Gibson M. A comparison of laparoscopy and laparotomy for the treatment of ectopic pregnancy. Obstet Gynecol 1988;71:889-92.

18. Silva P D. A laparoscopic approach can be applied to most cases of ectopic pregnancy. Obstet Gynecol 1988;72:944-7.

19. Pouly J L, Mahnes H, Mage G, Canis M, Bruhat M A. Conservative laparoscopic treatment of 321 ectopic pregnancies. Fertil Steril 1986;46:1093-7.

20. Lundorff P, Hahlin M, Kallfelt B, Thorburn J, Lindblom B. Adhesion formation after laparoscopic surgery in tubal pregnancy: a randomized trial versus laparotomy. Fertil Steril 1991;55:911-5.

21. Lund J. Early ectopic pregnancy: comments on conservative treatment. J Obstet Gynaecol Brit Emp 1955;62:70-6.

22. Dwarakanath L S, Mascarenhas L, Penketh R J, Newton J R. Persistent ectopic pregnancy following conservative surgery for tubal pregnancy. Br J Obstet Gynaecol 1996;103:1021-4.

23. Stromme W B. Conservative surgery for ectopic pregnancy: a twenty year review. Obstet Gynaecol 1973;41:215-23.

24. Maymon R, Shulman A, Halperin R, Michell A,Bukovsky I. Ectopic pregnancy and laparoscopy: review of 1197 patients treated by salpingectomy or salpingostomy. Eur J Obstet Gynaecol Reprod Biol 1995;62:61-7.

25. Dubuisson J B, Morice P, Chapron C, De Gayffier A, Mouelhi T. Salpingectomy- the laparoscopic surgical choice for ectopic pregnancy. Hum Reprod 1996;11:1199-1203.

26. Vermesh M, Silva P D, Sauer M V, Vargyas J M,Lobo R A.Persistent tubal ectopic gestation. Patterns of circulating beta-human chorionic gonadotrophin and progesterone and management options. Fertil Steril 1988;50:584-8.

27. Tulandi T, Guralnick M. Treatment of tubal ectopic pregnancy by salpingotomy with or without tubal suturing and salpingectomy. Fertil Steril 1991;55:53-5. 